\title{
Supplementary File of the TPDS Manuscript: High-performance Computing Implementations of Agent-based Economic Models for Realizing 1:1 Scale Simulations of Large Economies
}

Amit Gill, Madegedara Lalith, Sebastian Poledna, Muneo Hori, Kohei Fujita, and Tsuyoshi Ichimura

Abstract-This document provides the details of the algorithms to improve the completeness of the TPDS article - "High-performance Computing Implementations of Agent-based Economic Models for Realizing 1:1 Scale Simulations of Large Economies".

Index Terms-Agent-based Economic Models, High-performance Computing, One-to-one scale simulations, Large economies, Scale-Free Graphs, Message Passing interface, OpenMP

- Amit Gill is with the Department of Civil Engineering, The University of Tokyo, 7-3-1 Hongo, Bunkyo City, Tokyo, 113-8654, Japan.

E-mail: gill@eri.u-tokyo.ac.jp

- Madegedara Lalith, Kohei Fuiita, and Tusyoshi Ichimura are with the Department of Civil Engineering and the Earthquake Research Institute, The University of Tokyo, 7-3-1 Hongo, Bunkyo City, Tokyo, 113-8654, Japan.

E-mail: lalith, fujita, ichimura@eri.u-tokyo.ac.jp

- Sebastian Poledna is with the International Institute for Applied Systems Analysis, A-2361, Laxenburg, Austria. E-mail:poledna@iiasa.ac.at

- Mипеo Hori is with the Research Institute for Value-Added-Information Generation, Japan Agency for Marine-Earth Science and Technology, 3173-25, Showa-machi, Kanazawa-ku, Yokohama-city, Kanagawa, 2360001, Japan.

E-mail: horimune@jamstec.go.jp 
Algorithm 1: Pseudocode of the naive_update_distr version of Update_cumulative_probability_ distribution()

Input : Vector, $\mathbf{o}^{r, s}$, of the active-sellers,

Index, $j$, of the sold-out seller, and

Vector, $\mathbf{P}^{r, s}$, of the cumulative probability distribution

Output: $N_{a}$ and updated vectors $\mathbf{o}^{r, s}$ and $\mathbf{P}^{r, s}$

$1 \mathbf{o}^{r, s}$.erase $\left(\mathbf{o}^{r, s} \cdot \operatorname{begin}()+j\right)$;

$2 N_{a} \leftarrow \mathbf{o}^{r, s}$.size () ;

3 Re-evaluate the probability $p_{i}^{r, s}$ for the remaining elements of $\mathbf{o}^{r, s}$

4 Generate a cumulative probability distribution vector $\mathbf{P}^{r, s}=\left\{P_{i}^{r, s} \mid P_{i}^{r, s}=\sum_{j=1}^{i} p_{j}^{r, s}\right\}$;
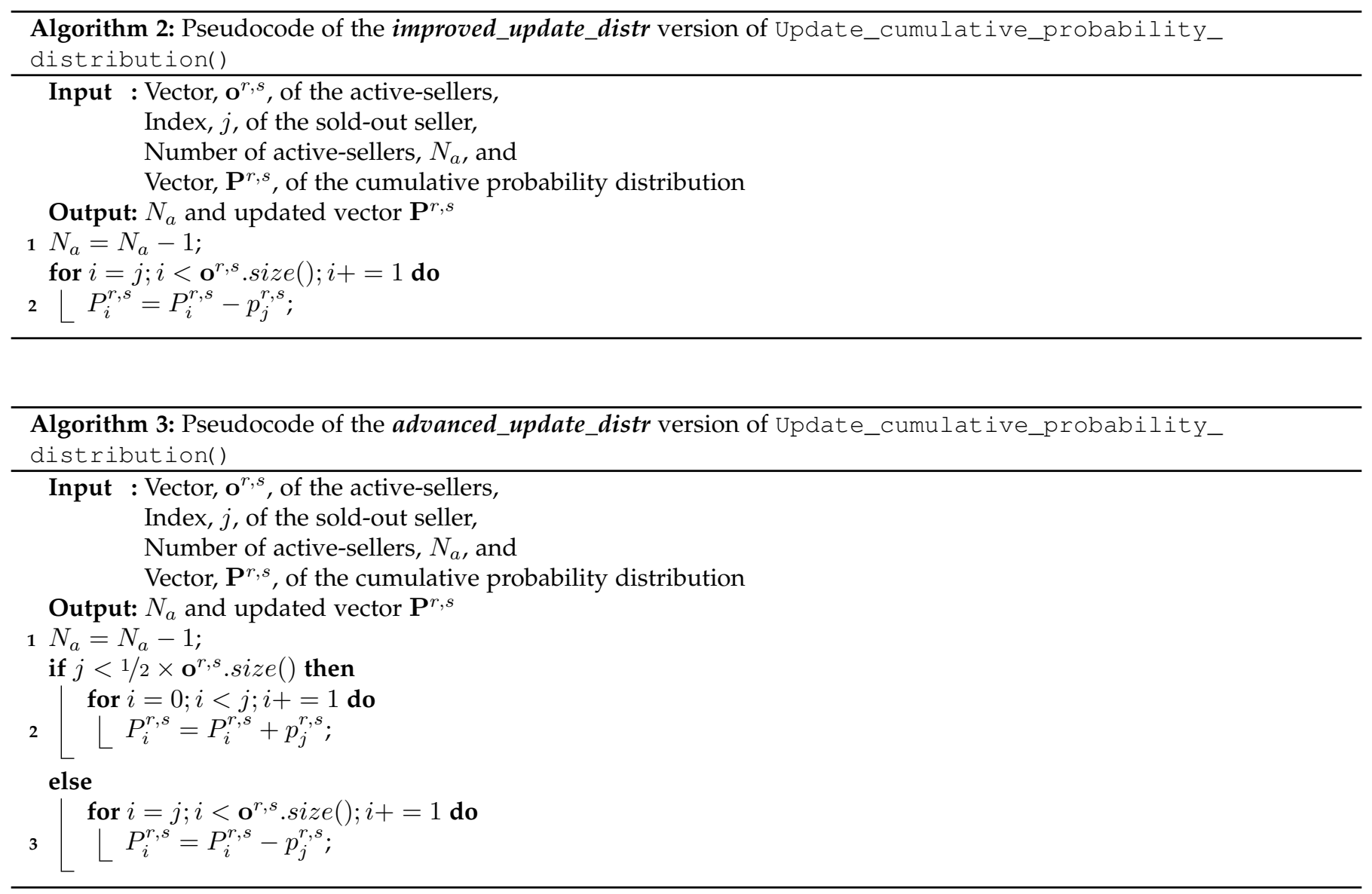


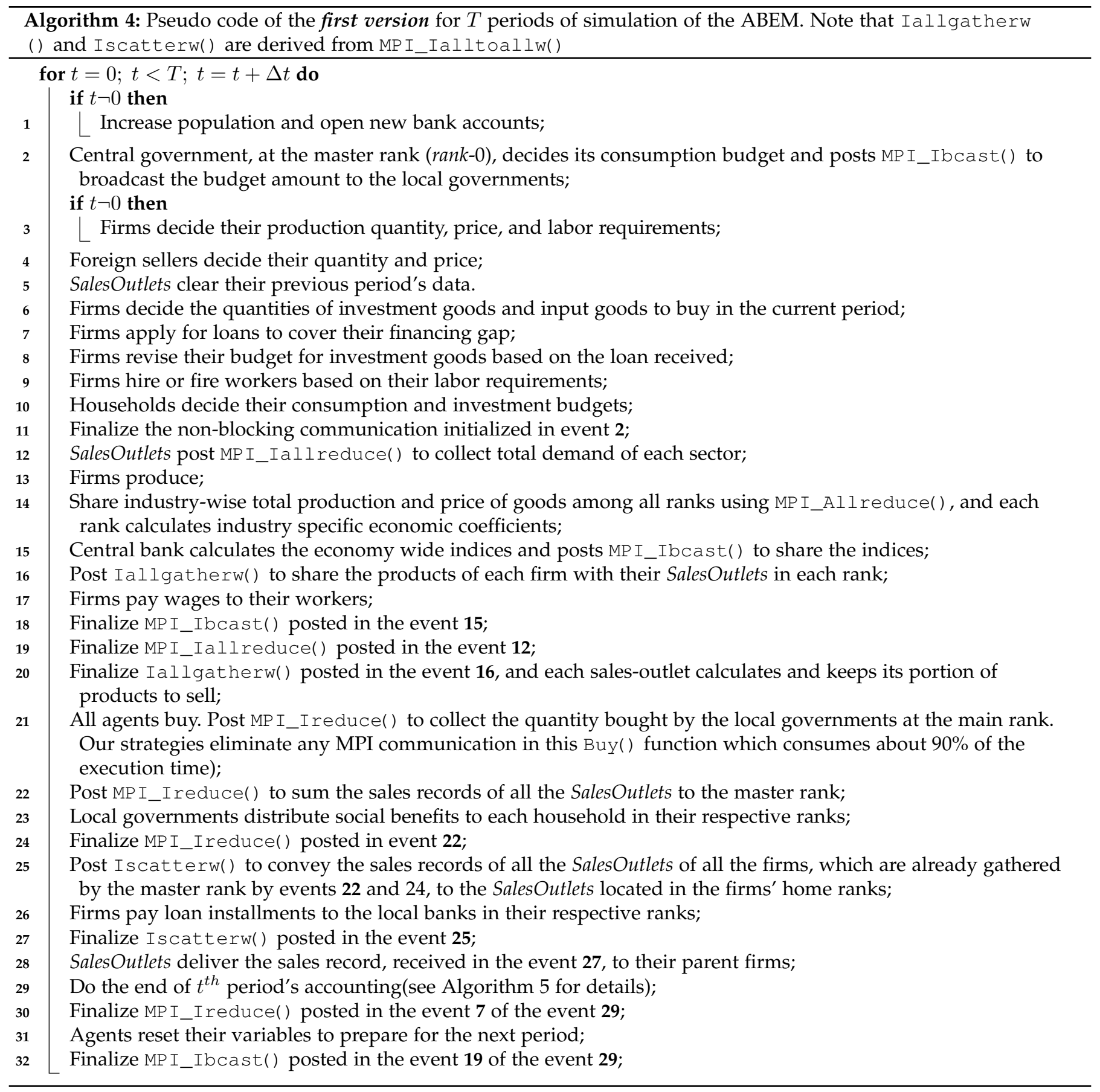




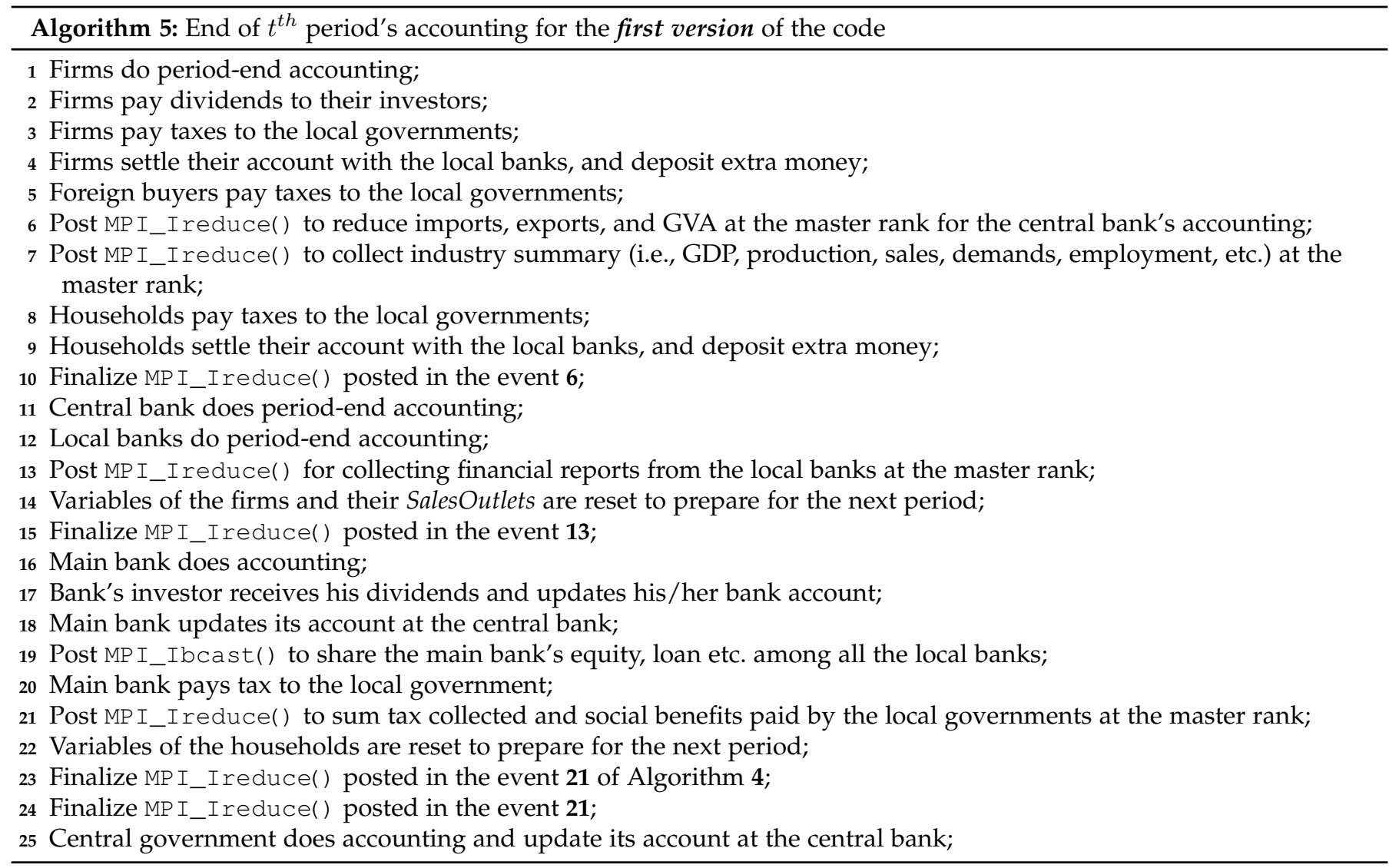




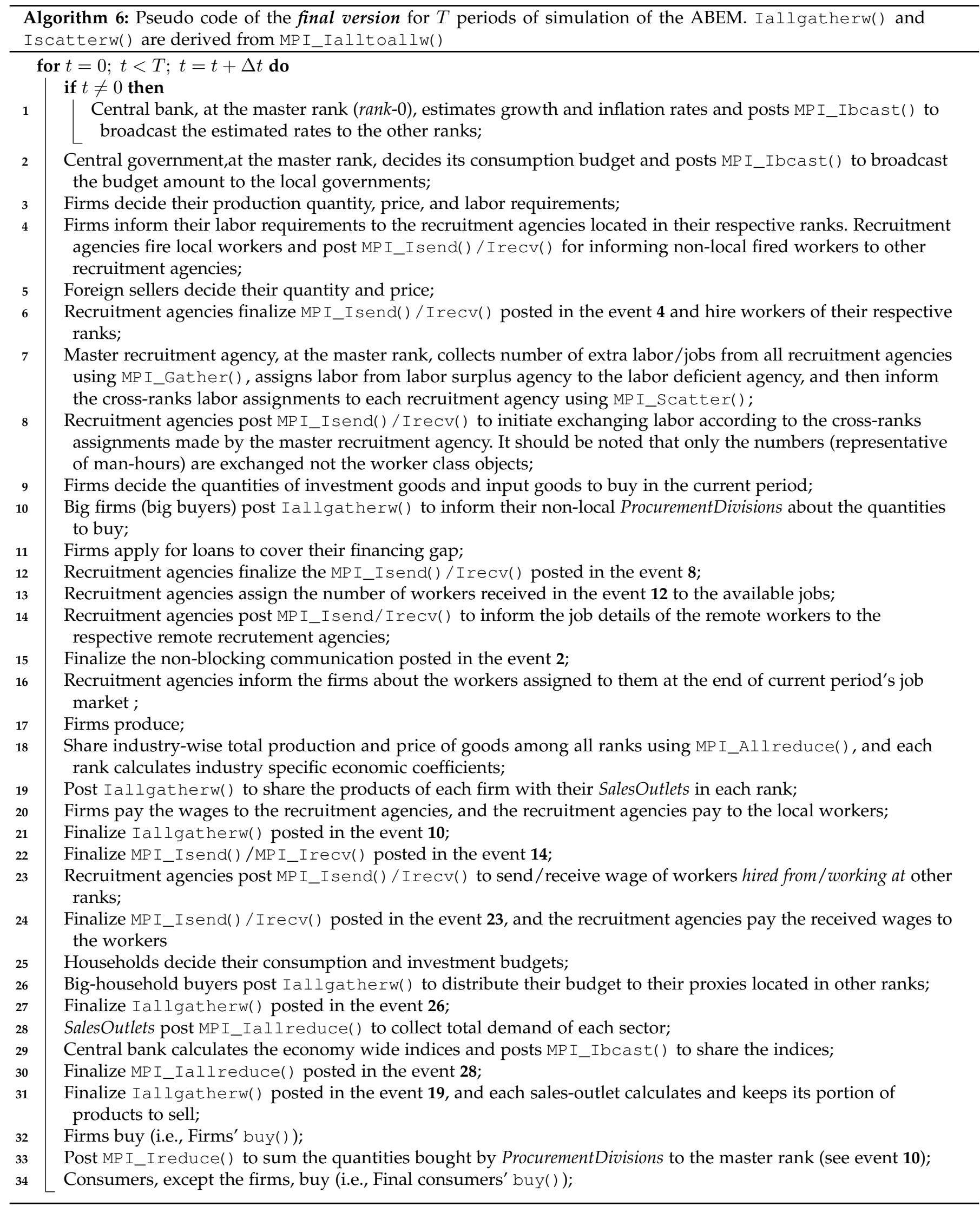


Finalize MPI_Ibcast( ) posted in the event 29;

Finalize MPI_Ireduce( ) posted in the event 33, and post Iscatterw() to convey the quantities thus gathered by the master rank to the ProcurementDivisions of the big firms located in their home ranks;

Post MPI_Ireduce( ) to sum the quantities bought by the proxies of big-household buyers to the master rank;

Post MPI_Ireduce( ) to collect the quantities of goods bought by the bank's investor;

Post MPI_Ireduce( ) to sum the sales records of all the SalesOutlets to the master rank;

Local governments distribute social benefits to each household in their respective ranks;

Finalize MPI_Ireduce() posted in the event 39, and post Iscatterw() to convey the sales records thus gathered by the master rank to the SalesOutlets located in the firms' home ranks;

Firms pay loan installments to the local banks in their respective ranks;

Finalize Iscatterw() posted in the event 41. SalesOutlets deliver the sales record, thus received, to their parent firms;

Finalize MPI_Ireduce( ) posted in the event 37, and post Iscatterw() to convey the quantities thus gathered by the master rank to the non-local buyers of the big household buyers located in the their home ranks;

Finalize Iscatterw() posted in the event 36. The ProcurementDivisions deliver the total quantity, thus received, to their parent firms;

Finalize Iscatterw( ) posted in the event 44. The non-local buyers deliver the total quantity, thus received, to their parent households;

Do the end of $t^{\text {th }}$ period's accounting (see Algorithm 7 for details);

Finalize MPI_Ireduce( ) s posted in the events 7 and $\mathbf{1 0}$ of the event 47;

Agents reset their variables to prepare for the next period;

Finalize MPI_Ibcast( ) posted in the event 20 of the event 47;

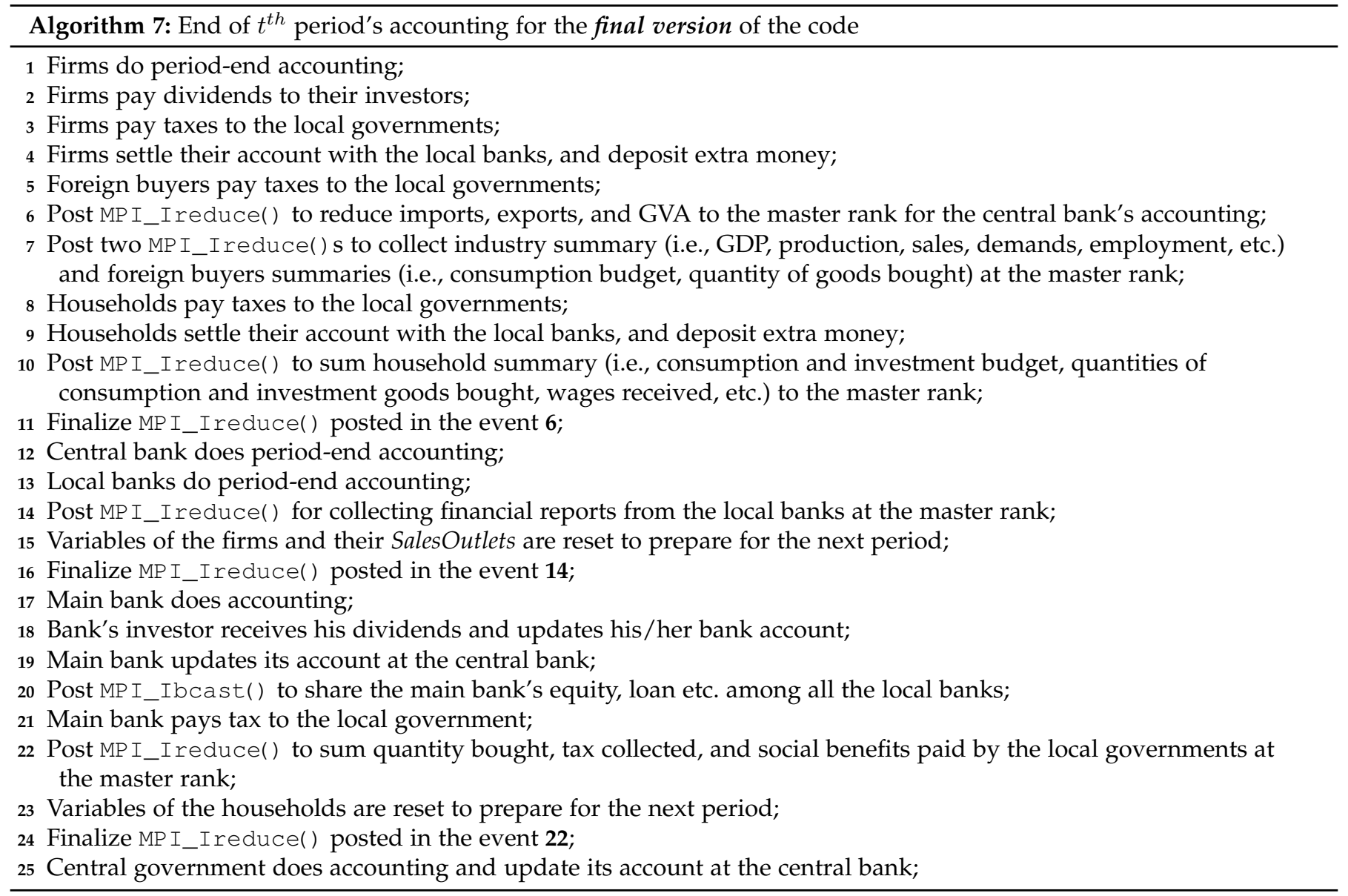

